\title{
On the Broader Sense of Life and Evolution: Its Mechanisms, Origin and Probability across the Universe
}

\author{
Melkikh $\mathrm{AV}^{1^{*}}$ and Mahecha $\mathrm{DS}^{2}$ \\ ${ }^{1}$ Ural Federal University, Yekaterinburg, Russia \\ 2Independent researcher, Bogota, Colombia
}

"Corresponding author: Melkikh AV, Ural Federal University, Yekaterinburg, Russia, Tel: +7-343-37593-49; E-mail: melkikh2008@rambler.ru

Received date: Sep 07, 2017; Accepted date: Sep 21, 2017; Published date: Sep 27, 2017

Copyright: (c) 2017 Melkikh A, et al. This is an open-access article distributed under the terms of the Creative Commons Attribution License, which permits unrestricted use, distribution, and reproduction in any medium, provided the original author and source are credited.

\begin{abstract}
We consider connection between the mechanisms of evolution of life and the existence of conditions suitable for life in the universe. In particular we review the problem of calculating the number of civilizations that might exist in the universe. We conclude that to solve this problem, the different mechanisms involved in the evolution of life should be taken into account in addition to well-known factors, such as mechanisms involved in star formation in galaxies, mechanisms leading to the self-destruction of civilizations, self-organization processes in planetary atmospheres and other factors. However, realistic times of existence and evolution of life (civilizations) can only be obtained under the assumption that evolution is partially directed. On this basis, a mechanism for the evolution of life in the universe- which contains the evolution of life on Earth as a special case- is proposed.
\end{abstract}

Keywords: Definition of life; Drake formula; Gaia effect; Partiallydirected evolution; Habitable zones; Biological complexity

\section{Introduction}

Hypotheses concerning the origin of life in the universe can be divided into several groups based on the literature $[1,2]$.

1. Life arose by chance on Earth but could occur many times in other regions of the universe. Once good conditions are created for life, then life occurs quickly.

2. Life is a unique phenomenon. The probability of its occurrence is vanishingly small. In the universe, there is only life on Earth.

3. The emergence of life and consciousness is one of the stages of the evolution of the universe (the strong anthropic principle).

4. The structure of the universe that we observe favors the emergence of life.

Unfavorable conditions in other universes cannot be observed because there are no observers (the weak anthropic principle).

However, of all these hypotheses, it is notable that the conclusion that is most adequate largely depends on the mechanism of biological evolution. Thus, the problem of the origin and evolution of life in the universe must be viewed in the context of models of the origin and evolution of life on Earth. Two of the main questions that must respond to the theory of evolution are as follows: What is the mechanism of the origin of complex systems? Can evolution explain the characteristic times of such systems (on Earth and in the universe)?

The emergence of life in the universe is an unsolved problem. On the one hand, the problem of the existence of conditions suitable to form life as we know it in the universe- and, in particular, on other planets- is not apparent. Many studies show that the Sun and the Earth have many special characteristics. In particular, to calculate the number of civilizations in the universe, the Drake formula [3-5] is often employed:

\section{$N=R \times f_{\mathrm{g}} \times f_{\mathrm{p}} \times n_{\mathrm{e}} \times f_{\mathrm{1}} \times f_{\mathrm{i}} \times f_{\mathrm{c}} \times L$}

where $\mathrm{R}$ is the mean star formation rate, $f_{\mathrm{g}}$ is the fraction of stars that can host planetary systems, $f_{\mathrm{p}}$ is the fraction of planetary systems that contain a habitable world, $n_{\mathrm{e}}$ is the average number of habitable worlds per system, $f_{1}$ is the fraction of habitable worlds that contain life, $f_{\mathrm{i}}$ is the fraction of inhabited worlds that contain intelligent civilizations, $f_{\mathrm{c}}$ is the fraction of intelligent civilizations that choose to communicate, and $L$ is the typical civilization lifetime.

Such a calculation based on the product is possible only when the subsequent steps are independent of the earlier steps. However, depending on the mechanisms of evolution (living systems, and the universe as a whole), these values may be part of a single process and therefore not independent of one another.

On the other hand, even if it is known that there are conditions suitable for life in its known form on a certain planet, it does not automatically mean that life will necessarily exist there. The origin and subsequent evolution of life is significantly determined by the mechanisms of its evolution. As shown, a partial directivity of evolution is a necessary factor to achieve the characteristic times on the order of billions of years of evolution [6-8]. Thus, a more detailed discussion of the factors $f_{1}, f_{\mathrm{i}}$ and their dependencies on the parameters must be undertaken.

The purpose of this article is to review the literature addressing the mechanisms of evolution and on the abundance of conditions suitable for life in the universe. It is important to discuss the possible connection between these two issues. This article is an attempt at a systems look at the evolution of life in the universe based on astrobiology, the theory of evolution (in the broadest sense), stochastic dynamics and cosmology.

\section{Stochastic dynamics and the probability of life}

At present, there are variety of approaches to calculate the probability of life by combining arbitrary quantities for some factors 
that are even too few to consider the development and sustainability of any type of life. Some methods include probability (Bernoulli) calculations, which exhibit independence between occurrences but also omit nonlinear complex dynamical systems that exhibit sensitive dependence to initial conditions.

Primordially, it is of great importance to consider system thinking to provide ground-breaking theories that explain and describe while interconnecting particular scientific fields. Otherwise, it makes the case for the lack of precision regarding the Drake formula, the mediocrity principle and calculations involving binomial distributions [9-11]. Even Darwinian theory, in which diversity- but not all the detailed sum of specificities and results from diverse scientific grounds- are taken into account. The linearity of the models [12] used to calculate the selection process excludes nonlinear dynamical complex systems with sensitive dependence on initial conditions. In addition, these models demonstrate that beyond the first generation, predictions cannot take place with accuracy. In fact, there is chaos and unpredictability in evolution at the phenotypical scale [13] and at the microscopic scales, e.g., there is complexity, non-extensivity and chaos in the DNA sequence of the Major Histocompatibility Complex [14]. There are uncountable changes that are not taken into account when nature's complex and systematic behaviors are modeled with linear (and even non-linear) models that exclude the richness and diversity of dynamic complex systems with sensitive dependence on initial conditions. Global and local speculations and conclusions appear as a result of a multi-scale level of inaccuracies, leading as in the case of Darwin's origin of species towards a global teleonomic approach from similar attributes, improvements, and ancestors not taking into account instead, similar but unique trajectories.

However, despite this apparent unpredictability, it will be shown that directivity in evolution can nevertheless take place.

The probability calculus and evolutionary process regarding the existence of life, must include deterministic, stochastic, self-similar, fractal and (last but not least important) chaotic behaviors. It is understood that such unique experimentation, simulation and calculation under the circumstances of sensitive dependence on initial conditions are an interesting approach regarding our uniqueness. We face uniqueness when such a paradox of repeatability under the same conditions yields different and countless particular results $[15,16]$. Chaotic phenomena challenge the traditional ways of regularity, uniformity, periodicity and countless classical reductionist approaches to nature's behaviors, resulting in a linearity operating from beginning to end on a universe that is mostly nonlinear when details are taken into account on a global basis. Such a complex sequence for the case about the appearance of life requires formulae that yield more precision to take into account spatiotemporal influences, nature's freedom, a priori information and the effects of any and all evolutionary processes. Such a sequence incorporates into the factors the possibility to include the physico-chemical history of evolution through probability, producing a calculation that leads to the conclusion that the probability of life in the universe tends to zero more than to one [16].

To accomplish such a detailed calculation, it is most important to first visualize the behavioral effects of the surrounding processes that lead to the emergence of life and even complex or intelligent life.

To construct such a sequence of calculations and a universal algorithm, a perfect set in topology is required that features self-similar iterations and the capability of providing different probabilities over time. The required set should be able to model the probability and the particularities of the formation path of every planet, and, in addition, the evolutionary path of life in the universe that takes into account the successes or failures of precise characteristics for the emergence and evolution of terrestrial planetary life. Specifically, the stochastic dyadic Cantor set (S.D.C.S.) with temporal and spatial randomness [17] addresses the characteristics that are required using an infinite number of intervals, infinitesimal size, existence near any point that belongs to the set itself, and a generating function with fractal properties on a [0, 1] interval that is not absolutely continuous and is also compact, perfect (every point is an accumulation point), and uncountable. This function provides a one-dimensional interval for calculating the sequential path of probabilities for every evolution of terrestrial planetary life in the universe- from its astronomical characteristics to the microscopic universe.

What follow, is a short introduction to the formulae that yields to better precision and that identifies the probability path of any terrestrial planetary formation to support life, higher life forms, or intelligent life through the S.D.C.S.

$$
P \text {, with, } 0<P<1
$$

\section{$t$, with, $t \geq 0$}

In the algorithm, $(\mathrm{Pt})$ represents the probability of each process, known or unknown, that did occur, is occurring or will occur through an interval of time within this framework of self-similar, stochastic, fractal and chaotic behaviors, where t (the time interval) is a range of time during which $\mathrm{P}$ remains constant. In other words, the constant probability of each process $(\mathrm{P})$ will have a time dependence $(\mathrm{Pt})$ on that interval of time $(\mathrm{t})$ in which it will be achieved or maintained through the iteration of the algorithm $\left(\prod_{t \geq 0}^{n}{ }^{*} P^{t}\right)$ and the precise characteristics and probability (PLife) to support life, higher life forms or intelligent life on a particular planet. $P_{\text {Life }}=\prod_{t \geq 0} P^{t}$

Notably, all outcomes derived using the S.D.C.S with temporal and spatial randomness are just as unique and desirable as planet Earth.

Through the course of various simulations, the tendency to zero is an invariant behavior that appears for different values for $t$ (time interval). The tendency to zero is the result of all possible combined probabilities of all occurrences that have appeared throughout the path before finding intelligent life [16].

Finally, the mechanism of the evolution of the protoplanetary cloud [18-20] implies that the process of planetary formation occurs throughout the universe and not just on particular planets. Through all processes that arise during planetary formation, the (PLife) of any type of life will appear as an emergent property with two primary and dichotomous characteristics [16]:

1. The (PLife) pattern appears with an infinite number of exact and precise arrangements at the micro and macro levels.

2. Such an expected (PLife) pattern will occur only by means of sensitive dependence on the initial conditions that will enable the necessary unique characteristics to arise, as exact precision is required. Infinite iterations are necessary to produce such an uncommon and fine-tuned outcome out of chaotic events. 


\section{Terrestrial planets in the universe}

Prior to the point of finding intelligent life, billions of years before it emerged, the formation process included chaotic behaviors [21] that were shaping future outcomes and configurations, such as the uniqueness of planet earth and of our solar system [16]. In addition, the presolar cloud collapse and the formation of the solar nebula exhibited the sensitive dependence on initial conditions [22].

From the plasma dynamics, there are multifractal behaviors consistent with the generalized weighted cantor set [23]. There is also gravitational instability on the dust to grain formation of planetesimals. In addition, magnetohydrodynamics (MHD) is a robust mechanism that interacts in the dynamic evolution of planets $[24,25]$, with its own chaotic pattern.

There is planetary migration with significant nonlinear mechanisms $[25,26]$ and the accumulation from planetesimals to protoplanets leads to an oligarchic growth that evolves into a chaotic system [27]. Of course, outcomes such as water contents out of these collisions are highly variable.

Chaos arises in systems with many degrees of freedom [28]. In the current solar system, there is chaotic evolution [29] and large-scale chaos [30]. The most immediate expression of this chaotic behavior is the exponential divergence of trajectories with similar initial conditions [31]. Thus, the Earth may experience a large chaotic zone from 0 to 85 degrees in its obliquity [31]. However, the moon causes the Earth to vary no more than 1.3 to 23.3 degrees in obliquity [31], which has already induced significant changes in insolation over the Earth's surface.

How typical is our solar system? The unlikely configuration of our solar system with such a variety of planets also relies on dynamic interactions that exhibit chaotic motions [31] and chaos over the spacing of the inner planets [32]. There are important considerations regarding advantageous circumstances that occurred for planets like Mother Earth. For example, the dynamics on the habitability of an earth-like planet with three terrestrial planets, will only work in low eccentricity orbits if there is a planet like Saturn with an inclination provided with less than 10 degrees. Otherwise, for inclinations greater than 20 degrees from a planet like Saturn, it is likely that one or several of the three terrestrial planets in the HZ (habitable zone) will escape [33]. If a planet remains orbiting without being ejected after such an event, high eccentricities would occur. It is important to note that high eccentricities indicate that- at some point- the planet orbiting the star in the $\mathrm{HZ}$ will become much closer and farther, being exposed to extremely high and low temperatures, which is not conducive for intelligent life.

Zackrisson and co-authors [34] evaluated the number of Earth-like planets in the universe. Such planets are understood to include planets of 0.5-2 times the Earth's radius. One of the important factors for the existence of Earth-like planets are the metallicity of the star, and the destruction of planets by gas giants. The likelihood of terrestrial planets existence in galaxies of different types was investigated. The authors suggest that perhaps we are the only civilization in our galaxy. However, to find extragalactic civilizations they are required to be powerful enough, which will be discussed in more detail in section 5 . As a result of these calculations, the total number of terrestrial planets is on the order of 1020 . It should be noted that as long as the mechanisms of evolution are not discussed, the exact number has little to tell us about the probability of the existence of life. For example, the rate of evolution can be limited by the enumeration of genome variants, the number of which is immeasurably greater. This issue will be discussed in discussion Section.

Hayrapetyan and co-authors [35] investigated the effects of solar flares in the early stages of evolution on prebiotic chemistry. One problem in this stage is the possible mechanisms of nitrogen fixation. The authors suggested that because the Sun was much more active during the early stages of its evolution than it is now, the impact of solar flares in the Earth's atmosphere meant that nitrogen began to engage in a variety of reactions. As a result, substances important to life were created. In the absence of such flares, nitrogen reactions might have been difficult. Thus, the authors' proposed mechanism may be important to the evolution of extrasolar planets orbiting Sun-like stars.

Recent photos of Pluto [36] suggest that it may contain water in a liquid state. If so, this fact may change our understanding of the possible presence of liquid water in relatively small bodies far from the Sun. Obviously, this fact would also extend the habitable zone around other stars because liquid water is one of the most important preconditions for life.

According to one study, Titan may be a promising location for life, despite its rather extreme conditions [37]. Authors of computer-based models have shown that the hydrocyanic acid formed in the atmosphere of Titan can react with other molecules and create Titan molecular chains or polymers such as polyimine. At the current temperature on Titan, polyimine can have a number of properties that might contribute to the development of living cells. Researchers believe that Titan polyimine can take multiple spatial configurations, and its ability to absorb sunlight will allow polyimine to produce energy for the development of life.

Frank and Sullivan [5] estimated a lower bound for the probability of the existence of technological civilizations in the universe. To this end, the authors have proposed to reduce the Drake formula to its two most important multipliers:

\section{$\mathrm{A}=\mathrm{N}_{\mathrm{ast}} \mathrm{f}_{\mathrm{bt}}$}

Where A is the number of all the technologically advanced species who have ever existed in the observable universe; $\mathrm{N}_{\text {ast }}$ denotes the number of potentially habitable exoplanets in a given part of the universe; and $\mathrm{f}_{\mathrm{bt}}$ represents the likelihood of developing a technological civilization on this planet. As a result, astrophysicists have concluded that human civilization is the only technologically advanced civilization in the visible universe only if the probability of the development of technological civilization in suitable for this exoplanet is $2.5 \times 10^{-24}$. According to these authors, the possibility of establishing contact with other civilizations is small enough because they are either already extinct or have not had time to achieve a sufficiently high level of development. On the scale of our galaxy, the authors assessed the probability of the development of other possible species on a potentially habitable exoplanet as 1: $60 \times 10^{9}$.

Thus, analysis of the possible number of planets in the universe, the conditions of which might be similar to Earth's, shows that this calculation is affected by and determined by many factors. An important role is played here by the chaotic motion of the planets and other parameters. The uncertainty in the knowledge of these factors does not permit accurate calculations; nonetheless, estimations on the order of magnitude are possible, and these estimations indicate that the number is in the range of $10^{20}$ planets. Note, however, that the presence of a planet with suitable conditions does not mean that there 
is necessarily life (particularly intelligent life) on that planet, as noted by other authors. In other words, such calculations give us, in fact, an upper estimate for the number of inhabited worlds because these estimations provide necessary but not sufficient conditions for life. To actually use the Drake formula, we must consider mechanisms of evolution with the most common positions. On the other hand, the very factors that affect the number of habitable worlds may result from deeper laws that also require more detailed consideration.

Note also that the concept of habitable zone is closely connected with the definition of life; thus, estimates of the probability of the existence of life in the universe essentially depend on what is meant by the term "life".

\section{Definitions of life and mechanisms of its evolution}

For a comprehensive review of the question of the probability of life in the universe, we must discuss the most common properties of living systems, including the most common mechanisms and their evolution. Such properties of systems such as homeostasis, information, algorithm, replication, reception, and evolution, among others, are the most common and largely do not depend on the specific physical and chemical form of the living system. The systems approach to biology in general, as well as to evolution in particular, involves the use of biological cybernetics for modeling biological systems. One of the most important concepts of evolution is the replicator- the simplest system capable of self-replication.

Many papers are devoted to unsolved problems regarding the origin of life [38-43].

Generally speaking, there may be alternative forms of life. A number of such possible forms based on silicon, arsenic and other elements have been considered in the literature [44].

Different definitions of life have been formulated repeatedly [45-47] although one sufficiently accurate determination is difficult to agree upon. For example, Tirard and co-authors [45] noted that over the most recent 18-20 centuries, scientists have mainly used a phenomenological description of the life, not the exact definition. Strictly speaking, an exact definition is only possible in mathematics. These authors thus posit that there are currently many definitions of life, but there is still no good one.

Note that biogenesis is clearly an interactive phase transition due to quantum computing of molecular kinetic energetics, which depends of the exact boundary conditions imposed by the bio-elements and the earth like scenario. This may lead towards a directed evolution, which should not be consider in the sort of an intelligent design hypothesis, which is also out of the scope of the present article. Thus, the genetic organismic evolution considered in the terms of directed evolution would be even more controversial in those terms. However, the genetic evolution is also a quantum phenomenon, which implies a causal relationship between information at the micro scale and the information in the natural system or mesoscopic scale. As stated, biogenesis is clearly, an interactive phase transition due to quantum computing of molecular kinetic energetics that depends of the exact boundary conditions imposed by the bio-elements and the earth like scenario.

Woolf [48] considered the question of the origin of biology and its differences from chemistry. According to this author, enzymes are an essential part of biology because they carry out specific work on the synthesis, transport, and catalysis of the most important life processes.
This author further noted three paradoxes associated with the emergence and evolution of the early stages of life:

\section{- A genetic paradox}

- An autocatalytic paradox (Muller paradox)

- The paradox of the emergence of biological complexity.

According to this author, the solutions to the first two paradoxes are thus associated with an intermediate stage in the evolution of life- the world of RNA. The solution to the third paradox is one of principal, as it involves both defining and understanding the mechanisms of its functioning and evolution. This issue will be discussed in more detail below.

Janković [49] believes that the laws of nature are not conducive to life. However, if life one way or another appears, with it arise mechanisms for its preservation. This author pays great attention to the concept of evolvability, considering it one of the most important to understanding life. Evolvability can be defined as the ability to evolve in a changing environment [49]:

Evolvability of a biosphere is the measure of summary potential of evolutionary change of all its living beings, together with some measure of overall propensity of its systems to undergo evolutionary change upon given conditions.

This property of living systems may evolve based on the second type of selection processes [50].

According to Janković [49], evolutionary changes are random in that they are not directed in advance for some purpose. However, they are not completely random because they use previous structures. This author considers the example of the evolution of Darwin's finches and believes that the paradox of time for them can be solved by the fact that the process of evolution is not just brute force but instead also a cooperative process using previously existing beaks. The principal, however, is to determine the mechanisms of a "partially random" evolution.

One of the manifestations of the concept of evolvability according to Janković [49] may be a change of the entire biosphere to increase the capacity of species to survive. This assumption is largely similar to the concept of Gaia, which is discussed below. The author believes that the information is playing an active role in evolution and proposes to consider the information as the basis of a working definition of life. An important role of information and coding for an understanding of life is also discussed in several other papers [51,52].

Melkikh and Seleznev [53] discuss the processes of transporting substances in the protocells. Transporting substances based on the biochemical structure of molecules is one of the simplest and most important properties of a living system. In particular, the regulation of the transport of substances can be used to maintain the intracellular concentration of a particular substance. One of the simplest materials in a transport control system can be represented as follows Figure 1: 


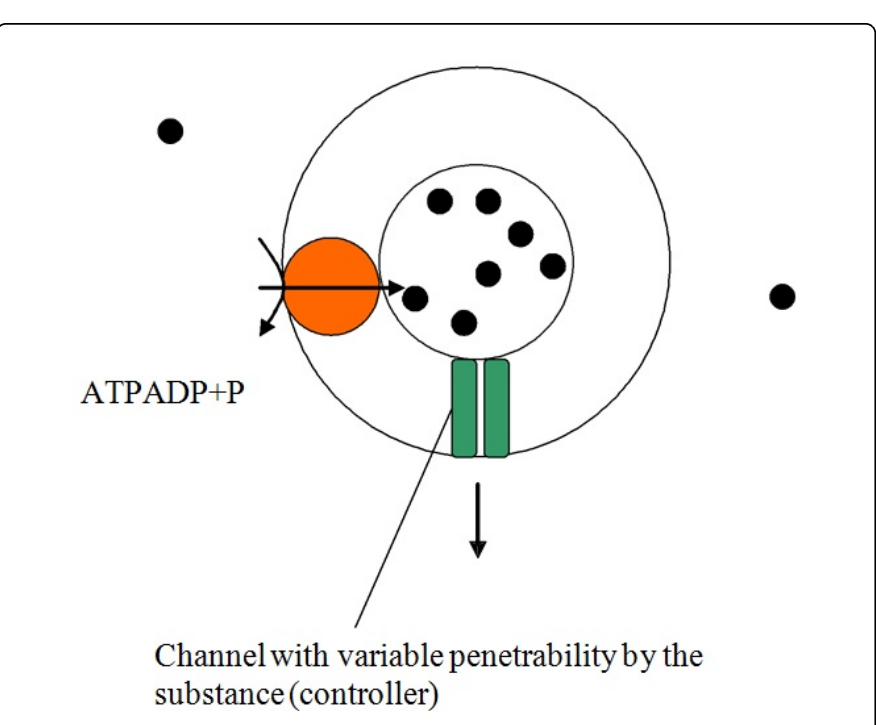

Figure 1: The simplest system of regulation of substance transport in a cell.

This system could work because of the non-equilibrium radiation of the stars and because of various chemical reactions [54]. In the work of Melkikh and Seleznev [53], it was proposed that there is at least one control system, which can be included in the definition of life. Further development of the application of control ideas to transporting substances in protocells and their movement was undertaken by Melkikh and Chesnokova [55] and Melkikh and Sutormina [56].

In various papers, Melkikh $[6,7,57,58]$ considers the problems of biological complexity in connection with the origin of life. The first problem is associated with the folding of biologically important molecules. It has been shown that the existence of a plurality of spatial structures of biologically important molecules- and many variants of chemical reactions between them- is one of the most important obstacles for the occurrence of the simplest living system. Melkikh [7] also concluded that there must be some special mechanism that greatly limits the range of variants in such a system. Without this mechanism, we cannot speak about any significant effectiveness of molecular machines in the protocell. Thus, this protocell will consist of various types of molecular complexes with different spatial configurations, and such complexes are unable to perform useful work. As the complexity of cells increases as evolution progresses, this problem will only worsen as the number and length of biologically important molecules increases.

The second problem is the problem of enumeration of exponentially large numbers of variants of the information sequence. This problem becomes acute when some molecules became encoded by other molecules. In particular, for a chain of length $\mathrm{N}$ with four types of nucleotides there are $4^{\mathrm{N}}$ possible variants. For example, for $\mathrm{N}=1000$ we obtain: $4^{1000} \approx 10^{602}$.

This number of variants is so great that it could not be enumerated for the lifetime of the universe for all organisms (replicators) that ever lived in it. However, $\mathrm{N}=1000$ corresponds to approximately only one modern gene. Hence, Melkikh [7] concluded that for information sequences of lengths equal to approximately 103 and larger, the enumeration of variants occurred in another way. The main question is: How and under what conditions will it work?
It is crucial to determine what the mechanisms of evolution should be that can be used to solve this combinatorial problem. It is believed that further evolution can be dictated by molecular exaptation, i.e., by dicing and using existing information sequences, blocks, etc. However, according to Melkikh [7], such a mechanism will not operate on its own, as it implies a priori information in the system to perform its function. Indeed, if the system has no prior information about how it will encode a set of characters, then there's no way to know about it but to synthesize a molecular machine on this information and check whether such an organism will survive (or not). This is a simple enumeration of variants. If a priori information is available, it should not simply be implied but instead have some material carrier, i.e., it should be recorded in some (not yet known) intracellular structures.

Another argument that is discussed in Paradoxes of early stages of evolution of life and biological complexity [7] is associated with general algorithms proposed for solving different classes of search problems. If, in the above situation (i.e., with no a priori information), an algorithm for solving the problem in a polynomial (relatively small) number of steps, exists, then it just would have been resolved as all other NP-hard problems. However, reducing the NP-algorithm (nonpolynomial) to the P-algorithms (polynomial) today remains unsolved.

The fundamental question arises: is it possible to speed up the search process in the absence of a priori information using any other process (molecular exaptation, horizontal gene transfer, alternative splicing, emerged later etc.)? The answer to this question [7] is negative- either a change in the information sequence occurs randomly or there must be a system that in some way selects nucleotides and performs certain operations with them. In the second case, the presence of a priori information in the system is a prerequisite for its operation.

Thus, according to Melkikh [7], the enumeration of variants paradox is this: of course, the molecular mechanisms of exaptation, block coding, horizontal gene transfer, etc., work (and could exist, apparently, during the early stages of evolution), but a priori information (which exists before the synthesis molecular machines) is indispensable to their operation. The modern theory of evolution does not imply any such information. Assuming that this information exists, the question arises, where is it stored because the information should have a physical carrier. On the other hand, information regarding genes changing cannot be stored in the genes themselves. Thus, the issue of such information storage and processing mechanisms remains open.

Formulated paradoxes are an obstacle to the emergence of life in any form (including alternative forms of life based on other chemical elements such as silicon, arsenic, etc.). Most likely, we are talking about the most common constraints that relate to all the known elements of the periodic table.

On the basis of the paradoxes formulated, Melkikh [7] gives the following definition of life: Life is a non-equilibrium self-reproducing system of such large complexity that in it cannot be implemented in a simple search of variants.

The above paradoxes entail the need to discuss alternative mechanisms of evolution. This issue will be discussed below. 


\section{The Gaia hypothesis and macroscopic correlations in biospheres}

In the context of the number of inhabited worlds, the Gaia hypothesis occupies an important place. According to this hypothesis, the conditions on a planet can change significantly as a result of life, i.e., life can provide the conditions for itself.

In the 1970s, Lovelock put forward the Gaia hypothesis [59], which posits that the set of all biological organisms is so intimately related to the evolution of their physical environment at the global scale that integrally they form a single self-developing system with selfregulating properties that resemble the physiological properties of a living organism. Being a super-organism, Gaia maintains some parameters of the internal environment at a relatively stable level that is favorable for living organisms. Recently, the emphasis in this field has changed slightly. The majority of scholars do not regard the Earth as a single organism. Concurrently, the altruistic or mutually beneficial behaviors of species (parts of the biosphere) remain the subject of wide speculation. For example, microorganisms, together with plants, accelerate rock decay thousands of times, resulting in self-regulation of the carbon dioxide cycle [59]. One of the mechanisms for this climate control is adjustment of the cloud canopy through the emission of sulfides by algae, etc.

Despite some superficial similarity of the biosphere to an individual organism, there are significant differences between them. In particular, the Gaia theory has been criticized primarily because the biosphere as a whole cannot offer an analog for DNA [60,50]. This discrepancy means, in turn, that it is impossible to formulate the mechanism for the Darwinian evolution of a biosphere, including the transfer of genetic information and selection.

In his papers, Staley [61] notes, that there are problems with the Darwinian interpretation of the model. One problem involves the fact that Darwinian adaptation destroys planetary correlations. The author has proposed a model which, in his opinion, does not contradict Darwinism and simultaneously provides for global regulation.

We can agree that there may be special cases that provide homeostasis and do not contradict Darwinism. However, it is doubtful that the evolution of all species and the biosphere can be explained using this model. Staley [61] examines the specific case of the regulation of the planet's temperature. However, this regulation is not limited to living organisms. For example, such regulation may occur in the equilibrium of various phases of a substance that is present in the biosphere in considerable amounts. If there is equilibrium between the phases (solid and liquid or liquid and gaseous), then the temperature can be maintained automatically.

Different variants of Daisyworld are considered, for example, in papers by Ackland, Pujol, Kleidon and Wilkinson [62-66].

McDonald-Gobson and co-authors [67] examined the properties of different models of Daisyworld. In particular, the minimum assumptions for these models were considered, and the sensitivity of the evolution of small changes in parameters was analyzed.

Boyle and co-authors [68] examine symbiosis as a basis for homeostasis in the Daisyworld. These authors also note that there is a problem with the natural selection of these systems. As noted above, looking at the term "evolution" in the broader context (in the sense of the temporal behavior of the system of arbitrary nature) results in no reason to require the presence of selection because the mechanisms of evolution may be quite different.
The authors examined the example of symbiosis of fungi and cyanobacteria. As a result, a model was built on the basis of which the authors concluded that the existence of macroscopic homeostasis is possible.

Kleidon [69] examined the thermodynamic efficiency of the planet as a whole and developed the modern definition of the Gaia hypothesis:

Hypothesis: The Earth system has evolved further and further away from a state of thermodynamic equilibrium in time mostly by the increased generation rate of chemical free energy by life.

In addition to global regulation performed by organisms over the entire planet, such regulation and variants of correlations (although macroscopic) are possible on a smaller scale as well. Marinakis [60] considered the analogy between protocells and plant (bacterial) communities and suggested that plant communities can be regarded as a phenotype of a microorganism. However, the problem of storing and transmitting information arises in such a system. Moreover, the quantum correlations between atoms of the macrosystem have been considered as possible alternatives to storing information [60,70]. However, this raises the question of how such a system could arise. In this case, the problem with Darwinian justifications for the origin of such a system remains because there is no competition against which such a structure could win or lose.

Chopra and Lineweaver [71] examined the model, which they called Gaian Bottleneck. According to these authors, in order to a planet was truly habitable, living beings on it should be able to control greenhouse gases and to maintain a stable temperature on the surface. In the evolution of planets, life in its simplest forms might play a significant role not only in the formation of the atmosphere but also in the formation of rocks and the movement of lithospheric plates. The authors believe that such mechanisms should occur quickly enough in the first billion years of life on the planet, which may explain why we have not yet been confronted with the "brothers on reason" or just have not found traces of life on other planets. Life just became extinct on most of them that were unable to control the climate and geology of the planet. Thus, the authors propose a solution to the Fermi paradox that, based on the fact that life, even if it is fairly widespread as the simplest forms in the universe, is vulnerable and has no time, in any way significantly, to alter habitat.

Thus, the Gaia hypothesis is useful to employ to consider the problem of the proliferation of life in the universe because it makes it possible to analyze the conditions under which some forms of macroscopic homeostasis may exist. Such macroscopic manifestations can be observed using modern devices, which means that we must be prepared in any way to interpret the data on extrasolar planets. Gaia models just play this role. However, as noted by many authors, the Gaia model will not work by itself, for its operation requires specific conditions. A discussion of these conditions is important in the broader context.

Is such a homeostasis at the scales of the galaxy and the universe possible?

\section{Super-civilizations and their evolution}

Kardashev [72] created a scale of civilizations, in which it was suggested that there were several types of civilizations, including one that has the ability to exploit the energy of an entire galaxy. The characteristic time of existence of civilization represents one of the 
factors in the Drake formula. This is one of the parameters and is that with the most uncertain information. Nevertheless, it makes sense to discuss ways of its experimental verification, and the most common limitations on the use of energy civilizations.

Harrison [73] suggested that our universe was made by more advanced life forms from another world. In particular, the values of the fundamental constants can be obtained by selection. The hypothesis of selection among the worlds has been discussed in other studies [74].

Although the spread of civilization in the space (material, energy, etc.) is generally accepted, there is yet another point of view. According to Smart [75], the evolution of civilization may develop in the opposite direction (transcension hypothesis). The author compares the evolution of living systems (in particular, its aspect of evo-devo) to the evolution of civilization. This comparison is important and will be discussed below in the context of the theory of partially directed evolution. In particular, this author believes that the civilization is advanced enough to be away at small sizes, with the result that they will not be visible. The author believes that this may explain the Fermi paradox. Recall that this paradox posits that if there is a sufficiently large number of extraterrestrial civilizations, then at least some of them should already be fixed but that this has not yet occurred.

As a result, the supercivilization that comes to a considerable complication of the structure in small volumes will have its process's ultimate state as a black hole, according to this author. The author believes that at this stage, the evolution of civilization may develop quite controllably and predictably.

What are the signals indicating the existence of extraterrestrial civilizations that may be registered on Earth? This issue is the subject of a series of articles. Carrigan [76] discussed the possibility of detecting Dyson spheres in our and other galaxies. Currently, there are several candidates for this class of radiation sources. It is expected that traces of civilizations will also register on the spectra of artificial elements, such as technetium and plutonium. It may be added that a sufficiently advanced civilization must be able to synthesize and use many of the elements of the periodic table of the island of stability. Natural mechanisms of the synthesis of such elements with long-lived enough charge numbers that are greater than 130 are not known.

Detection of Dyson spheres are also discussed in other articles [77,78]. In particular, a number of authors discuss the notion supercivilization use energy of pulsars. In particular, Osmanov [78] suggested that in the case of pulsar, which emits enough energy into a narrow beam, Dyson "sphere" will no longer be a sphere, but a ring. Ring sizes are discussed depending on the properties of the pulsar. It was concluded that the rapid pulsars require far too much material for the ring.

Chennamangalam and co-authors [79] suggested that the pulsar signal can be modulated with the help of satellites to send signals to other civilizations.

To solve the Fermi paradox, Stevens [80] considered the duration of the existence of civilization. In his opinion, this is the greatest factor limiting the total number of existing civilizations. In this limit, only one civilization (ours) may be in the world because the others have died quickly. This author has considered various causes of civilizations' self-destruction, which are as follows:

Nuclear war

Chemical and Biological degradation
Technological disaster

\section{Global pollution}

The results of nuclear war on exoplanets are possible to register from Earth on the basis of changes in the chemical composition of their atmospheres, as well as by gamma radiation. Authors have discussed various ways of registering other types of global change on a planet.

However, it is impossible to exclude the possibility that these methods are programmed strategies by which the total number of civilizations in the universe is maintained at a certain level.

Another solution to the Fermi paradox was proposed by Solomides and Terzian [81]. According to these authors, the absence of signals from aliens and visible traces of life in the surrounding space speaks in favor of the notion that intelligent life dwells at a distance of at least fifteen hundred light-years from Earth and will not be found before the corresponding time. In contrast to the rare Earth theory [2], these authors considered Earth as an ordinary planet, the conditions on which could be frequently and regularly met in the galaxy. According to the calculations of those authors studying the field of communication with the Earth, the number of planets is too small to conclude anything about the prevalence of life in the universe. The basis of the calculations is the assumption that life, once it has arisen on some planet, will inevitably become intellectual. However, as noted above, there are strong arguments against the proposition that life in its higher forms was widespread [2].

Gurzadyan [82] considered possible forms of information transmission both for intelligent signals and for travels of extraterrestrial life. The author concluded that life propagate in the universe not via files containing the information on them, but the programs- coded strings defined by Kolmogorov complexity. If this is true, then we need new methods in the studies of the cosmic signals, and can eventually approach the solution to the Fermi paradox.

The problem of detecting extraterrestrial civilizations is addressed by Cabrol [83]. The author believes that all the prerequisites for an interdisciplinary approach to the problem have now been assembled. The author believes that connecting our knowledge with that of planetary and space sciences, astrobiology, life sciences, cognitive sciences (in conjunction with communications theory), bioneural computing, machine learning and big data analysis is required. There is no doubt that this interdisciplinary approach will contribute to the search for extraterrestrial civilizations, assuming they exist. However, the same can be said about the problem of the existence of life in the universe- estimating the likelihood of its existence is also necessary to unite the various sciences.

One of the extreme points of view in this context is the one, in which not only super-civilizations exist but also our entire world is a computer simulation controlled by such super-civilizations [84-86]. This point of view might, to some degree, answer the question posed by Wigner $[87,88]$, "Why is mathematics effective?" Beane and coauthors [86] considered the limitations on computational resources required for the calculation of the fundamental interactions within the cellular automata. These authors suggested that the above scenario of a computer simulation may appear in the distribution of high-energy cosmic rays, which could reflect the properties of the lattice on which the simulation takes place.

The work of Tegmark [85] is an attempt to prove that our world is not just a computer simulation but the mathematical structure. 
However, it is most likely that such a statement cannot be confirmed or disproved by experiment.

\section{Anthropic principle or physical law?}

The anthropic principle is that the fact that we as observers exist imposes certain restrictions on the properties of the world that we observe. There are various formulations of the anthropic principle. In particular, the weak anthropic principle states that observers may exist in the universe with certain parameters. The strong anthropic principle states that the parameters of the universe are such that a biosphere will inevitably form in the future, including one containing human civilization.

Waltham [89] used a Bayesian approach to anthropic selection of the properties of inhabited planets. According to Waltham [89], the distribution of the different properties of the planets must be different for the inhabited and uninhabited planets. For example, planets with an unstable climate are less likely to gain an observer than a planet with a stable climate. The author believes that the Gaia hypothesis is not justified in the sense that climate stability is necessary for the existence of complex life but not vice versa. The author concludes that planets with Earth-type biodiversity must be rare in the universe.

A number of properties of space objects can be explained on the basis of the anthropic principle. Iorio [90] obtained limits on the cosmological constant based on the assumption that life exists. These impose restrictions on this value from bottom and top. In particular, with a certain value of the cosmological constant is the position of the Sun in the galactic habitable zone. Another example is the assessment of the concentration of dark matter using anthropic considerations by Freivogel [91]. Perhaps there is the influence of dark matter in the long-period comets, which, in turn, affect the mass extinctions of organisms on Earth [92].

Considering various variants of the anthropic principle in connection with the problem of supersymmetry, Kane [93] opposed the directivity of evolution of the universe on the basis that, in any case, accidents such as falling asteroids that could destroy life, etc. are inevitable. In other words, the universe is not so well- adapted for life. However, this objection is unfounded, as any directed processes in nature and technology contain accidents. Their repeated duplication of processes, etc. provides for the elimination of accidents. In other words, an accident can be a natural part of the directed evolution, both within the ecosystem and within the universe.

Other examples of the application of the anthropic principle to the structure and properties of the universe were considered [94,95].

Does a multi-world in which the fundamental constants have different values exist? This issue has been addressed many times, but it is not the purpose of this article to address it again. Analyzing various cosmological models is also not our goal.

Thus, some of the properties of the universe (and its individual parts) can be explained by the presence of observers; however, such a posteriori explanation cannot be considered final. It is important to understand the physical laws that explain the properties of the universe a priori. This approach is the basis of all the natural sciences.

\section{Discussion: Hypothesis of directed evolution}

Review of the problem of the emergence, evolution and prevalence of life in the universe allows us to come to the following conclusions:
The formation of the planets and their further movement inevitably contains a chaotic element that significantly reduces the likelihood that life even as it is emerged will be able to evolve to the level of an advanced civilization.

Although the number of habitable planets in the universe is estimated to be in a fairly wide range- approximately 1020 (with differences of several orders of magnitude), but this quantity is not the greatest barrier to the existence of civilizations (the factor in the Drake formula). The speed of the evolution of life is a much more complex problem that requires exhaustive search.

Macroscopic correlations (such as the Gaia model) are possible on a scale of planets, but for their existence, life must have certain properties. These properties themselves do not follow from the evolution, as controlled by selection and random mutations.

Part (but not all) of the properties of planets, galaxies and other cosmic structures can be explained on the basis of the anthropic principle, which entails the need to build a unified theory of the evolution of the universe at different levels of the hierarchy.

A clear definition of life is currently missing. Most likely, not only a definition but an understanding of the processes of its functioning and evolution is necessary. The most fundamental are the laws covering the origin and evolution of complex structures.

Generally speaking, civilization, even arisen, may be unstable with respect to many factors. Their short lifespans will significantly reduce the number of currently existing civilizations.

It is obvious that all of these various factors require coordination, forming a single testable theory regarding the origin and evolution of life in the universe.

To solve combinatorial problems in papers a model of partially directed evolution was proposed $[6-8,57,58,96]$. The term "partially" reflects the fact that the uncertainty will remain present in the environment in some form, even if evolution might be completely directed, which is due to the thermal motion of the molecules, climate uncertainty, random events such as asteroid strikes, etc. The main provisions of the model of partially directed evolution are as follows:

- Evolution is a priori directed (in this it is similar to the morphogenesis)

\section{- Selection and random mutations play a secondary role,}

- Storage and processing of a priori information obeys to the quantum laws. Justification of the latter provision is contained in the works and connected with the contradictory of alternatives- the classic method of a priori information storage [6-8].

The proposed model of partially directed evolution allows us to solve a combinatorial problem because in it enumeration of variants area is substantially limited a priori, i.e., enumeration occurs among the knowingly known "good" variants. Of course, errors may occur in the process of enumeration, which leads to the partial directivity of evolution.

One of the main properties of directed evolution (in light of the combinatorial problems solution) is that evolution is directed from the beginning (from the early stages). This property allows us to naturally assume that these very early stages of evolution resulted from partially directed processes occurring in the universe before the origin of life. 
The following interpretation of anthropic principle variants discussed above can be given from the perspective of the theory of partially directed evolution. The weak anthropic principle is a natural consequence of the diversity of natural conditions. In the same manner and in the biosphere, the probability of finding an observer in the zones with a favorable climate, generally speaking, is larger than in the Arctic wilderness, for example. However, in this embodiment, the anthropic principle does not answer questions regarding the mechanisms of evolution on Earth and in the universe.

A much more fruitful and verifiable approach is the one according to which the evolution of the universe (including life in it) is a natural process. It is possible that life arises naturally at a certain stage of the universe. Such ideas have been expressed in the past [1], but the mechanisms of this evolution must be clarified.

In what sense can we understand the strong anthropic principle? It can be understood as a consequence or a different formulation or partially directed evolution on the scale of the universe. The literature has repeatedly considered the fine-tuning of the universe in other words, the dependence of the conditions of the existence of life on fundamental constants and other parameters of physical interactions [97-99]. As shown below, the theory of partially directed evolution on the scale of the universe has testable consequences have been proposed by Melkikh [6]. Therefore, this principle can be understood as a physical law. This physical law does not require the introduction of the "goal" concept for the evolution of the universe. There are many physical laws (e.g., the laws of classical mechanics), which, being deterministic, are unrelated to the purpose of motion.

Two factors can be distinguished in the state of the universe that are most important to its evolution and to further the origin of life. One factor is the fundamental constants and physical laws themselves, in which the constants are included. As has been shown previously, their value must be in fairly narrow ranges for the universe had the type that we observe $[97,98]$. Fundamental constants are most likely only understood as necessary conditions. With their "adverse" values, life will not arise, but these specific values by themselves do not lead to the emergence of life.

The most important factor for the evolution of life in the universe may be the second factor - the initial conditions. In the early stages of the big bang, the universe had certain parameters: the coordinates and velocities of the particles (taking into account the uncertainty principle) as well as the characteristics of the fields. More specifically, the solution to the complete system of equations describing the evolution of the universe requires the specification of the wave functions of particles and fields at the initial time. Those initial conditions, in conjunction with physical laws and constants, defined the values that would completely determine the evolution of the universe and life in it.

According to modern theories, there are two main variants of the evolution of the universe: inflation and a "big bounce" [100,101]. In the first case, the universe emerged from nothing at some point of time, which can be considered the initial, with its rapid expansion at the initial stage (inflation). In the second case, the expansion of the universe came after the stage of strong compression. The purpose of this article is not a detailed analysis of the merits and demerits of various cosmological models. It should be noted, however, that the big bouce variant is more preferable, since it does not violate the conservation laws. This variant is also more preferable with respect to the evolution of life, since the emergence of complex living systems "from nothing" cannot be considered as the basis of evolution theory.

According to various models of big bounce, the state of the universe after expansion is in one way or another related to the state before expansion [101-103]. From the point of view of the emergence of life, the approach associated with non-Archimedean dynamics seems promising [104]. This approach is based on the assumption that, at high densities, the interaction between particles undergoes a significant change- the triangle inequality is violated. This entails a significant change in the laws of particle dynamics. Thus, the state of the universe at the early stages of its expansion is encoded in the parameters of non-Archimedean dynamics and in the parameters of the universe at the compression stage.

The complete system of wave functions of the initial time (после начала расширения) provides information on its subsequent evolution. It is not necessary that this information can be calculated by von Neumann's formula. However, the behavior of a quantum system is completely determined by its initial conditions and the system of equations describing the evolution of particles and fields.

Part of such a priori information is the information on the base of which at some point living systems begin to arise. This information corresponds to the degrees of freedom of particles and fields, which in the early stages of the universe's expansion could not manifest themselves in any way. The first step, in which this information is beginning to play a significant role, perhaps, is the emergence of replicators with a specific three-dimensional structure. As shown earlier, the first problem in the early stages of evolution is precisely the problem of the folding of replicating molecules [6].

Is the assumption that the wave functions of the particles at the initial time were quite certainly contrary to known laws of physics? Most likely not. In models of the universe, its state at the initial time is often considered as the average and is characterized by values such as pressure, energy density, etc. As noted above, it is possible that the initial distribution of the particles is simply the result of the universe's previous evolution [105].

Further evolution of particles and fields in an expanding universe depends on the expansion model. There are a number of models that assume a permanent expansion and a subsequent contraction of the universe. Several authors have previously proposed models based on which the selection may occur among universes by some mechanism $[73,74,106]$. Such a selection would result in a certain initial state of our universe. However, is it possible to check the possibility of whether multi-world existence is problematic and extends beyond science.

Based on the foregoing, the hypothesis of directed evolution of life in the universe can be formulated in the form of the following provisions:

1. In the initial moment of the big bang, physical constants, in addition to the wave functions of particles and field properties, took (as a result of some unknown previous evolution) the certain values.

2. These specific values of constants and fields have led to the fact that the macroscopic parameters of the universe have evolved such that in the future stars and galaxies formed, the value of the curvature of space (as well as other parameters of the universe) that contributed to (creating the necessary conditions) the emergence of life on a later stages. 
3. At a certain stage, in the presence of these necessary conditions after the emergence of atoms and molecules in certain systems, quantum laws have begun to play an important role and to control the spatial structure and evolution of replicators. Ultimately, these laws were determined by the initial state of the universe.

4. In the future, such partially directed evolution follows in the direction of the complexity of organisms, increasing their adaptation to different environmental conditions. The laws of partially directed evolution are ultimately the result of the laws of physics and the initial conditions that characterize the universe. This evolution can be considered a new physical law ("the program of evolution").

5. Macroscopic correlations in the biosphere (Gaia effect) may be a natural consequence of partially directed evolution, as such mechanisms don't require Darwinian selection and random mutations. In this sense, the evolution of the biosphere is a separate part of the directed evolution of the universe.

\begin{tabular}{|l|l|}
\hline $\begin{array}{l}\text { Evolution of the universe, } \\
\text { including the evolution of life }\end{array}$ & $\begin{array}{l}\text { Morphogenesis and behavior of } \\
\text { organisms }\end{array}$ \\
\hline Billions of years. & Hours, years. \\
\hline $\begin{array}{l}\text { Determined by the initial conditions } \\
\text { and by the world's constants; } \\
\text { however, chaos and accident also } \\
\text { play important roles. }\end{array}$ & $\begin{array}{l}\text { Determined by genes; however, } \\
\text { environment also plays an important } \\
\text { role. }\end{array}$ \\
\hline $\begin{array}{l}\text { The lifetime of the universe is } \\
\text { limited? }\end{array}$ & The lifetime of an organism is limited. \\
\hline $\begin{array}{l}\text { Life in the universe arises at a } \\
\text { relatively late stage. }\end{array}$ & $\begin{array}{l}\text { Some organs (properties of behavior) } \\
\text { appear at later stages (for example, } \\
\text { flowers in plants). }\end{array}$ \\
\hline $\begin{array}{l}\text { Compensatory mechanisms must } \\
\text { exist to ensure that the evolution of } \\
\text { life in the universe occurs when } \\
\text { there is destruction of life } \\
\text { (civilization) in some parts of the } \\
\text { universe. There must be alternative } \\
\text { (replacement) life forms for extreme } \\
\text { conditions. }\end{array}$ & $\begin{array}{l}\text { There are compensatory mechanisms } \\
\text { (variants of behavior) that begin to work } \\
\text { when environmental properties deviate } \\
\text { from optimal values. }\end{array}$ \\
\hline
\end{tabular}

Table 1: Similarities and differences between the evolution of the universe (including the evolution of life in it), on the one hand, and morphogenesis and behavior of organisms, on the other.

The proposed hypothesis, first, does not require any new physics (at least at this stage). Second, this hypothesis is testable. One of the consequences of the proposed hypothesis might be that the calculation of the number of civilizations in the universe cannot be made on the basis of the production of probabilities- values in Drake's formula, generally speaking, are not independent and are themselves part of a united process- of the partially directed evolution of the universe.

On the other hand, it is also necessary to emphasize that the proposed hypothesis does not allow for estimating the likelihood of life or the number of inhabited worlds directly. This hypothesis itself requires a separate check, and on this basis, it will be possible to estimate the probability of the origin of life anywhere in the universe.

In accordance with the above observation, an analogy can be drawn between the morphogenesis and behavior on the one hand and with the evolution of the universe, including the evolution of life, on the other (Table 1).
Notably, the behavior (in particular, the acquisition of knowledge when we consider intelligent systems) is a process that is largely similar to evolution. In particular, Melkikh $[107,108]$ demonstrated that the acquisition of knowledge leads to a contradiction, which means that all animal and human behavior programs are innate. This process is a natural part of the overall process of the directed evolution of life in the universe.

In this connection, it is appropriate to revisit the issue that was discussed in paragraph 5: why our understanding of the universe is at least to some extent true? Why is it knowable and simulated, for example, in the language of mathematics? Mathematics can be considered as one means of controlling the universe (some compensatory property) on the basis of a priori information. The universe is correctly described by mathematics because mathematics is based on the same priori information on which this very universe is built, which is why mathematics is sometimes effective even when the experimental possibilities remain far from possible to verify its assertions.

Thus, the hypothesis proposed allows for explaining the evolution of life on Earth and in the universe from the same uniform positions.

Consider some of the experiments that might confirm or refute the hypothesis of directed evolution of life or its individual provisions. A number of such experiments were also proposed by other authors.

Naturally, the most informative experiment involved attempting to detect other (including earlier) forms of life in the universe. Such an experiment is not possible now, but there may be different types of experiments related to the consequences of the proposed hypothesis.

One of the ways to test the hypothesis might be experimentally to study the early stages of the universe's evolution. Studying the properties of cosmic microwave background radiation (as well as gravitational waves and other signals) might provide information regarding the degree to which the state of particles and fields in the early stages of evolution has been ordered. A number of these experiments was proposed by Hameroff and Penrose [105,109] to check his ideas, but they may also give us information on proposed hypothesis. Studying the properties of dark matter and dark energy might provide information about some of the feedbacks, with the help of which certain properties of the galaxies and the universe as a whole adjust.

Another possible direction is the study of exoplanets. At present, the study of exoplanets is intensely developing. In particular, the statistics of change of planets' atmospheres could provide information on the distribution of the effects of the Gaia hypothesis- the macroscopic correlation in the planet's atmosphere, which cannot be explained by the action of physical laws not related to the activity of organisms. The detection of small changes in the composition (movement) of the planets can be an argument in favor of this hypothesis.

Finally, direct observation of quantum effects (in the folding of molecules, replication, recognition) in the evolution of life on Earth can be considered to argue in favor of the proposed hypothesis. For example, if the experiments on the rapid (over several generations) evolution of a species show its directivity, it will be a direct confirmation of the hypothesis. Such evolutionary experiments were discussed earlier $[6,8,110]$ in more details. 


\section{Conclusion}

The origin and evolution of life in the universe is determined by many factors, many of which will inevitably contain random components. At the same time, to assess the possible number of civilizations in the universe (or inhabited planets), it is important to discuss the various mechanisms of evolution. In particular, when solving combinatorial problems exponentially, small probabilities arise, which are many orders of magnitude smaller than when taking into account other factors (e.g., the randomization of the planetary orbits, the instability of planetary atmospheres). The solution to the combinatorial problems is possible in the framework of the hypothesis of partially directed evolution. This hypothesis naturally generalized to the partially directed evolution of the universe in which the origin of life at a certain stage is natural.

Between morphogenesis, behavior of the organisms, on the one hand, and the evolution of the universe (including the evolution of life), on the other analogies can be carried out. The existence of such analogies is not random but may result from the community of the laws of evolution at different levels of the organization. Experiments to test the hypothesis of partially directed evolution were also considered.

\section{References}

1. de Duve C (2011) Life as a cosmic imperative? Phil Trans R Soc A 369: 620-623.

2. Ward PD, Brownlee D (2000) Rare Earth, Copernicus, New York.

3. Drake F (1962) Intelligent Life in Space. MacMillan, New York.

4. Walters C, Hoover RA, Kotra RK (1980) Interstellar colonization, a new parameter for the Drake equation? Icarus 41: 193-197.

5. Frank A, Sulivan WT (2016) A New Empirical Constraint on the Prevalence of Technological Species in the Universe. Atrobiology 16: 359-362.

6. Melkikh AV (2014) Quantum information and the problem of mechanisms of biological evolution. BioSystems 115: 33-45.

7. Melkikh AV (2015) Paradoxes of early stages of evolution of life and biological complexity. Orig Life Evol Biosph 45:163-171.

8. Melkikh AV, Khrennikov A (2016) Quantum-like model of partially directed evolution. Prog Biophys Mol Biol 125: 36-51.

9. Burchell MJ (2006) W(h)ither the Drake equation? Int J Astrobiology 5: 243-250.

10. Kukla A (2009) Extraterrestrials, A Philosophical Perspective. Lexington Books.

11. Rossmo DK (2016) Bernoulli, Darwin, and Sagan, the probability of life on other planets. Int J Astrobiology 16: 185-189.

12. Lande R, Arnold S (1983) The measurement of selection on correlated characters. Evolution 37: 1210-1226.

13. Ispolatov I, Doebeli M (2014) Chaos and unpredictability in evolution. Evolution 68: 1365-1373.

14. Pavlos GP, Karakatsanis LP, Iliopoulos AC, Pavlos EG (2015) Measuring complexity, non-extensivity and chaos in the DNA sequence of the Major Histocompatibility Complex. Physica A 438: 188-209.

15. Berliner M (1992) Statistic, Probability and Chaos. Stat Sci 7: 69-90.

16. Mahecha DS (2016) Evolution through the stochastic dyadic Cantor set, the uniqueness of mankind in the universe. Int J Atrobiology 15: 319-331.

17. Hassan MK, Pavel RK, Kurths J (2014) Dyadic Cantor set and its kinetic and stochastic counterpart. Chaos, Solitons \& Fractals 60: 31-39.

18. Safronov VS (1967) The protoplanetary cloud and Its Evolution. Soviet Astronomy 10: 650-658.

19. Kuchner MJ (2004) A minimum-mass extrasolar nubula. The Astrophysical J 618: 1147-1151.
20. Setiawan J, Henning Th, Launhardt R, Müller A, Weise P, et al. (2008) A young massive planet in a star-disk system. Nature 451: 38-41.

21. Diacu F, Holmes P (1997) Celestial encounters, the origins of chaos and stability. Princeton University Press, Princeton, NJ.

22. Boss AP, Goswami JN (2006) Presolar Cloud collapse and the formation and early evolution of the solar Nebula. In Meteorites and the Early Solar System II. D. S. Lauretta, H. Y. McSween Jr. (eds.), (University of Arizona Press, Tucson), 943, pp: 171-186.

23. Macek WM (2009) Chaos and multifractals in the Solar System Plasma. In Topics on Chaotic Systems. Eds. Skiadas C.H., Dimotikalis I., Skiadas C. Singapore, 214-223.

24. Balbus SA, Hawley JF (2000) Solar nebula magnetohydrodynamics. Space Sci Rev 92: 39-54.

25. Rafikov RR (2004) Fast accretion of small planetesimals by protoplanetary cores. Astron J 128: 1348-1363.

26. Clement M, Kaib N (2017) Prevalence of chaos in planetary systems formed through embryo accretion. Icarus 288: 88-98.

27. Nagasawa M, Thommes EW, Kenyon SJ, Bromley BC, Lin DNC, et al. (2007) The diverse origins of terrestrial-planet systems. In Protostars and Planets V. B. Reipurth, D. Jewitt, and K. Keil (eds.), University of Arizona Press, Tucson, 951, pp: 639-654.

28. Beauge C, Callegari N Jr, Ferraz-Mello S, Michtchenko TA (2005) Resonances and stability of extra-solar planetary systems. Dynamics of populations of Planetary Systems Proceedings IAU Colloquium 197.

29. Sussman GJ, Wisdom J (1992) Chaotic evolution of the solar system. Science 257: 56-62.

30. Laskar J (1994) Large scale chaos in the solar system. Astron Astrophys 287: 9-12.

31. Laskar J (2003) Chaos in the solar system. Ann Henri Poincar 4: 693-705.

32. Laskar J (1997) Large scale chaos and the spacing of the inner planets. Astron Astrophys 317: L75-L78.

33. Pilat E (2015) The role of dynamics on the habitability of an earth like planet. Int J Astrobiology 14: 145-152.

34. Zackrisson E, Cassilendorf P, Gonzalez J, Benson A, Johansen A, et al. (2016) Terrestrial planets across space and time. Astrophys J.

35. Airapetyan VS, Glocer A, Gronoff G, Hebrard E, Wanchi W, et al. (2016) Prebiotic chemistry and atmospheric warming of early Earth by an active young Sun. Nat Geosci 9: 452-455.

36. Hammond NP, Barr AC, Parmentier EM (2016) Recent tectonic activity on Pluto driven by phase changes in the ice shell. Geophys Res Lett 43: 6775-6782.

37. Rahm M, Lunine JI, Ushber DA, Shalloway D (2016) Polymorphism and electronic structure of polyimine and its potential significance for prebiotic chemistry on Titan. PNAS 29: 8121-8126.

38. Hansma HG (2014) The power of crowding for the origins of life. Orig Life Evol Biosph 44: 307-311.

39. Luisi PL (2014) A new start from ground zero? Orig Life Evol Biosph 44: 303-306.

40. Ikehara K (2014) [GADV]-Protein World Hypothesis on the Origin of Life. Orig Life Evol Biosph 44: 299-302.

41. Wills PR, Nieset K, McCaskill JS (2015) Emergence of coding and its specificity as a physico-informatic problem. Orig Life Evol Biosph 45: 249-255.

42. Saha R, Pohorille A, Chen IA (2014) Molecular crowding and early evolution. Orig Life Evol Biosph 44: 319-324.

43. Himbert S, Chapman M, Deamer DW, Rheinstadter MC (2016) Organization of nucleotides in different environments and the formation of pre- polymers. Sci Rep 6: 31285 .

44. Rampelotto PH (2010) The Search for Life on Other Planets: SulfurBased, Silicon-Based, Ammonia-Based Life. J Cosmol 5: 818-827.

45. Tirard S, Morange M, Lazcano A (2010) The definition of life, a brief history of an elusive scientific endeavor. Astrobiology 10: 1003-1009.

46. Margulis L, Sagan D (1995) What is Life? Weidenfeld and Nicholson, London. 
47. Joyce GF (1994) Foreword. In Origins of Life, The Central Concepts, Eds, D.W. Deamer and G. Fleischaker, Jones and Bartlett, Boston, 1994.

48. Woolf NJ (2015) A hypothesis about the origin of biology. Orig Life Evol Biosph 45: 257-274.

49. Janković S (2016) Evolvability is an evolved ability, the coding concept as the arch-unit of natural selection. Orig Life Evol Biosph 46: 67-79.

50. Dawkins R (1986) The blind watchmaker, New York, Penguin.

51. Yockey HP (2000) Origin of life on earth and Shannon's theory of communication. Comput Chem 24: 105-123.

52. Yockey HP (2002) Information theory, evolution and the origin of life. Information Sciences 141: 219-225.

53. Melkikh AV, Seleznev VD (2008) Early stages of the evolution of life: a cybernetic approach. Orig Life Evol Biosph 38: 343-353.

54. Falkowski PG, Fenchel T, Delong EF (2008) The microbial engines that drive Earth's biogeochemical cycles. Science 320: 1034-1039.

55. Melkikh AV, Chesnokova OI (2012) Origin of the directed movement of protocells in the early stages of the evolution of life. Orig Life Evol Biosph 42: 317-331.

56. Melkikh Av, Sutormina M (2013) Developing synthetic transport systems. Springer Science \& Business Media.

57. Melkikh AV (2008) DNA computing, computation complexity and problem of biological evolution rate. Acta biotheoretica 56: 285-295.

58. Melkikh AV (2014) The problems of replication in the early stages of evolution: enumeration of variants and spatial configurations of replicators. Orig Life Evol Biosph 44: 283-285.

59. Lovelock J, Gaia A (1979) A new look at life on earth. Ox-ford University Press, Oxford.

60. Marinakis YD (2007) From quasi-organism to protolife. Ecological Complexity 4: 102-112.

61. Staley M (2002) Darwinian selection leads to Gaia. J Theoretical Biology 218: $35-46$.

62. Ackland GJ (2004) Maximization principles and daisyworld. J Theoretical Biology 227: 121-128.

63. Ackland GJ, Clark MA, Lenton TM (2003) Catastrophic desert formation in Daisyworld. J Theor Biol 223: 39-44.

64. PUJOL $\mathrm{T}$ (2002) The Consequence of Maximum Thermodynamic Efficiency in Daisyworld. J Theor Biol 217: 53-60.

65. Kleidon A (2002) Testing the effect of life on Earth's functioning: How Gaian is the Earth system.

66. Wilkinson DM (2003) Catastrophes on daisyworld. Trends Ecol Evol 18: 266-268.

67. McDonald-Gibson J, Dyke JG, Di Paolo EA, Harvey IR (2008) Environmental regulation can arise under minimal assumptions. J Theor Biol 251: 653-666.

68. Boyle RA, Lenton TM, Watson AJ (2011) Symbiotic physiology promotes homeostasis in Daisyworld. J Theor Biol 274: 170-182.

69. Kleidon A (2010) Life, hierarchy, and the thermodynamic machinery of planet Earth. Phys Life Rev 7: 424-460.

70. Davies P (2004) Does quantum mechanics play a non-trivial role in life? BioSystems 78: 69-79.

71. Chopra A, Lineweaver C (2016) The Case for a Gaian Bottleneck, The Biology of Habitability. Astrobiology 16: 7-22.

72. Kardashev NS (1964) Transmission of information by extraterrestrial civilizations. Soviet Astronomy 8: 217-221.

73. Harrison ER (1995) The natural selection of universes containing intelligent life. Q J R astr Soc 36: 193-203.

74. Smolin L (2006) The status of cosmological natural selection. arXiv,hepth/0612185v1.

75. Smart JM (2012) The transcension hypothesis: Sufficiently advanced civilizations invariably leave our universe, and implications for METI and SETI. Acta Astronautica 78 : 55-68.

76. Carrigan RA (2012) Is interstellar archeology possible? Acta Astronautica 78: 121-126.
77. Garrett MA (2015) Application of the mid-IR radio correlation to the G sample and the search for advanced extraterrestrial civilizations. Astronomy and Astrophysics 581: L5, 1-6.

78. Osmanov Z (2016) On the search for artificial Dyson-like structures around pulsars. International Journal of Astrobiology V.15, issue 2: $127-132$.

79. Chennamangalam J, Siemion APV, Lorimer DR, Werthimer D (2015) Jumping the energetics queue: Modulation of pulsar signals by extraterrestrial civilizations. New Astronomy V.34: 245-249.

80. Stevens A, Forgan D, O'Malley JJ (2015) Observational signatures of selfdestructive civilizations. International Journal of Astrobiology V.15, 4: 333-344.

81. Solomides E, Terzian Y (2016) A probabilistic analysis of the Fermi paradox.

82. Gurzadyan VG (2005) Kolmogorov complexity, string information, panspermia and the Fermi paradox. Observatory 352: 125-129.

83. Cabrol NA (2016) Alien midscapes - a perspective on the search for extraterrestrial intelligence. Astrobiology 16: 661-676.

84. Bostrom N (2003) Are we living in a computer simulation? The Philosophical Quarterly 53: 243-255.

85. Tegmark M (2008) The mathematical Universe. Foundations of Physics V. 28, issue 2: 101-150.

86. Beane SR, Davoudi Z, Savage MJ (2014) Constraints on the universe as a numerical simulation. Eur Phys J A 50: 148.

87. Wigner E (1967) The unreasonable effectiveness of mathematics in natural sciences. In Symmetries and Reflections. Indiana University Press, pp: 222-237.

88. Carter B (1974) Large number coincidences and the anthropic principle in cosmology. In Confrontation of Cosmological Theories with Observational Data, IAU Symposium No. 63, Reidel, Dordrecht, pp: 291-298.

89. Waltham D (2011) Testing anthropic selection, a climate change example. Astrobiology 11: 105-114.

90. Iorio L (2010) Anthropic constraints on the cosmological constant from the Sun's motion through the Milky Way. Monthly Notices of the Royal Astronomical Society 403: 1469-1473.

91. Freivogel B (2010) Anthropic explanation of the dark matter abundance. J Cosmology and Astroparticle Physics 03: 021.

92. Randall L (2015) Dark matter and the dinosaurs. Harper Collins, Ecco Press: 2015

93. Kane G (2013) Supersymmetry and Beyond. From the Higgs boson to the New Physics. Basic Books. New York.

94. Mack KJ (2011) Axions, inflation and the anthropic Principle. J Cosmology and Astroparticle Physics 07: 021.

95. Kizek M (2012) Dark energy and the anthropic principle. New Astronomy 17: 1-7.

96. Melkikh AV, Khrennikov A (2017) Molecular recognition of the environment and mechanisms of the origin of species in quantum-like modeling of evolution. Progress in Biophysics and Molecular Biology.

97. Davies P (2007) Cosmic Jackpot, Why Our Universe Is Just Right for Life. New York, NY, Orion Publications.

98. Rees MJ (1999) Just Six Numbers, Harper Collins Publishers.

99. Morison I (2013) Introduction to astronomy and cosmology. Hoboken, N.J., Wiley.

100. Weinberg S (2008) Cosmology. Oxford University Press, Oxford.

101. Bojowald M (2007) What happened before the big bang? Nature Physics 3: 523-525.

102. Bojowald M (2015) Quantum cosmology: a review. Reports in Progress in Physics 78: 023901 (21pp).

103. Ijjas A, Steinhardt PJ (2017) Fully stable cosmological solutions with a non-singular classical bounce. Physics Letters B 764: 289-294.

104. Dragovich B, Khrennikov AYu, Kozyrev SV, Volovich IV, Zelenov EI (2017) p-Adic Mathematical Physics: The First 30 Years. arXiv: $1705.04758 \mathrm{v} 1$. 
Citation: Melkikh AV, Mahecha DS (2018) On the Broader Sense of Life and Evolution: Its Mechanisms, Origin and Probability across the Universe. Astrobiol Outreach 6: 162. doi:10.4172/2332-2519.1000162

Page 13 of 13

105. Penrose R (2011) Cycles of time, an extraordinary new view of the universe. New York, Knopf.

106. Vidal C (2009) Fine-tuning, quantum mechanics and cosmological artificial selection. Foundations of Science 17: 29-38.

107. Melkikh AV (2014) Congenital programs of the behavior and nontrivial quantum effects in the neurons work. BioSystems 119: 10-19.

108. Melkikh AV (2014) The No Free Lunch Theorem and hypothesis of instinctive animal behavior. Artificial Intelligence Research V.3, No 4: 43-63.
109. Hameroff SR, Penrose R (2014) Consciousness in the universe. A review of the Orch OR theory. Physics of Life Reviews 11, issue 1: 39-78.

110. Melkikh AV, Khrennikov A (2015) Nontrivial quantum and quantumlike effects in biosystems, unsolved questions and paradoxes. Progress in Biophysics and Molecular Biology V.119, 2: 137-161. 